\title{
Formación de médicos especialistas en el Sistema de Servicios de Salud de Chile. Su evolución en el período 2007-2010
}

'Universidad de Chile.

Facultad de Medicina,

Departamento de Medicina. Campus Centro. Hospital San Borja-Arriarán

${ }^{2}$ Ministerio de Salud

División de Gestión y

Desarrollo de las Personas.

Departamento de

Formación y Capacitación

Recibido el 9 de septiembre de 2010, aceptado el 29 de abril de 2011

Correspondencia a: Dr. Oscar Román A.

Huelén 154 Depto. 2.

Prov. Santiago. Chile.

E-mail: or.alemany@gmail.

\author{
OSCAR ROMÁN A. ${ }^{1}$, MAURICIO FELIÚ R. ${ }^{2}$, LUIS A. ECHAVARRÍA ${ }^{2}$
}

\section{Training of specialists in the Chilean national health services}

Several institutions, such as the Ministry of Health, Universities, the Chilean Medical Association, Scientific societies and public opinion, recognize that there is a deficiency of specialized physicians in Chile. To overcome this shortage of specialists, the Ministry of Health, along with universities, is developing diverse initiatives to train specialists and cope with the requirements of the country. Seventy five percent of posts offered were filled by physicians. The number of positions increased from 173 in 2007 to 576 in 2010, with a cumulative total of 1582 physicians in four years. Fifty two percent are being trained in Basic Primary Specialties and $48 \%$ in primary specialties. Thirty three percent of graduates have the obligation to continue working in the public service during a certain lapse. This figure will increase to $50 \%$ in the following years. These specialists are mainly working in the more densely populated regions of the country. The universities that offer the higher number of training positions are the University of Chile, The Catholic University of Chile and the University of Santiago.

(Rev Med Chile 2011; 139: 762-769).

Key words: Education, medical, graduate; Health services; Medical specialties.
$\mathrm{R}$ ecientemente la prensa nacional, las autoridades universitarias, legislativas, del Ministerio de Salud y del Colegio Médico han reconocido el déficit de médicos especialistas en el país, principalmente en los hospitales del sistema público ${ }^{1-5}$. En este problema intervienen factores dependientes del sistema de salud, de las universidades formadoras y de los mismos actores médicos.

La formación de médicos especialistas depende de las escuelas de post-título de las universidades mediante programas de formación acreditados y debidamente certificados ${ }^{6}$. Los médicos que no han sido certificados como especialistas por las universidades han tenido la oportunidad de hacerlo rindiendo examen en la Corporación Nacional Autónoma de Certificación de Especialidades Médicas (CONACEM) ${ }^{7}$, entidad privada de derecho público, que reúne la participación conjunta de universidades, Academia de Medicina, Colegio Médico y sociedades científicas, en la cual el Ministerio de Salud (MINSAL) participa como observador.

La función asistencial de los especialistas en el sistema público de salud depende del ministerio del ramo, el que ha tenido un rol administrativo y financiero en la formación de ellos, a través de la Unidad de Formación de la División de Gestión y Desarrollo de las Personas ${ }^{8}$. Esta, en conjunto con la Asociación de Facultades de Medicina (ASOFAMECH), ha llevado a cabo un programa de formación de especialistas durante 4 años, iniciado en el año 2007.

De acuerdo a la información existente, se describirá el tipo de especialidades consideradas, la procedencia de los médicos reclutados, la distribución regional y el destino final de ellos en el sistema público o privado. 
Formación de médicos especialistas en Chile - O. Román et al

\section{Material y Método}

La División de Gestión y Desarrollo de las Personas del Ministerio de Salud y su Departamento de Formación y Capacitación ${ }^{9}$, ha establecido una relación estrecha con las universidades formadoras de post-título del país para realizar diferentes programas de formación de especialistas. Para ello, las universidades han ofrecido cupos de formación, que han sido debidamente financiados por el ministerio.

Los médicos ingresados a los programas de especialización desde el 2007 provienen de 4 líneas de contratación: médicos en etapa de destinación y formación (EDF), médicos pertenecientes a la Atención Primaria de Salud (APS) municipalizada, médicos comisionados directamente por los servicios de salud ${ }^{8,9}$ y el recientemente creado Programa de Formación de Especialistas Básicos para la APS, destinado a médicos recién egresados y que se contratan en consultorios municipales, alternando trabajo y formación por un período de seis años ${ }^{10}$. En este estudio no se han considerado los especialistas formados mediante financiamiento de las mismas universidades, de otras instituciones o incluso autofinanciados.

Los cupos disponibles de las universidades han sido ofrecidos a los médicos interesados, provenientes de las líneas funcionarias mencionadas, que los han ocupado de acuerdo a sus preferencias. Por tanto se produjo una diferencia entre los cupos ofrecidos y los realmente ocupados.

Los programas ofrecidos consideran las especialidades primarias básicas ( 5 en la actualidad, incluyendo Psiquiatría), las especialidades primarias y las subespecialidades de las primarias básicas $^{6,11}$.

Se ha realizado un registro acucioso del número de médicos recién egresados o contratados como médicos generales que ha optado por las especialidades madre (básicas) o por las primarias, su distribución regional, la obligación de retorno y el financiamiento general del sistema de formación.

\section{Resultados}

\section{Proceso de formación}

Hemos reconocido cuatro líneas programáticas de formación, a saber:
Becas Primarias. Instauradas en la década de 1950-59 para recién egresados, pagadas por el Estado, se desarrollaron hasta el año 2007. Duraban 3 años y los profesionales tenían posteriormente la posibilidad de continuar una subespecialización ${ }^{11}$. Una fórmula similar ha sido reinstaurada en el año 2008 con el objetivo explícito de reforzar la atención primaria municipal, deficitaria en la motivación de los médicos para ingresar a ella y también en su poder de resolución de los problemas médicos ${ }^{10,12}$. Las especialidades básicas que comprende dicho programa son: Medicina Interna, Pediatría, Cirugía General, Obstetricia-Ginecología y Psiquiatría. Duran 6 años y comparten períodos formativos en APS y atención secundaria y terciaria. Son impartidas por las universidades y están en desarrollo desde el año $2008^{10}$.

Médicos Generales de zona. Corresponden a los actualmente contratados por la Ley 19.664 en la primera etapa de la carrera funcionaria o etapa de destinación y formación ${ }^{8}$. El ingreso es por concurso nacional realizado por MINSAL. Inicialmente deben desempeñarse como médicos generales durante al menos 3 años en la APS en zonas geográficamente apartadas. Desde el tercer año pueden optar, también vía concurso nacional, a programas de especialización. Una vez finalizado éste, no tienen obligación de retorno al servicio público.

Médicos de atención primaria municipal. Se rigen por el Estatuto de Salud Municipal, y mediante concurso nacional pueden optar a programas de especialización por un período máximo de 3 años, teniendo obligación de retorno para el cumplimiento de un "período asistencial obligatorio" (PAO) por el doble del período de formación.

Médicos de formación directa por los servicios de salud (SS). Son médicos contratados por los servicios de salud, los que pueden optar, al cabo de 3 o más años, a una comisión de estudio para especializarse a través de cupos entregados por las universidades directamente a los servicios de salud. Tienen PAO con devolución del doble del tiempo de formación.

La gestión administrativa y presupuestaria de estas 4 líneas de especialización se realiza coordinadamente entre el Departamento de Formación 
y Capacitación del MINSAL y los Servicios de Salud, que otorgan el financiamiento necesario $y$ la Asociación de Facultades de Medicina (ASOFA$\mathrm{MECH}$ ), que ofrece anualmente cupos aportados por las universidades del país.

En la Tabla 1 se muestra el número de médicos ingresados a los programas según las líneas de formación. En la medida que transcurren los años entre 2007 y 2010, el número de ingresados aumentó en forma importante, llegando a un total de 1.600. En la Tabla 2 se anotan las diferencias entre los cupos ofertados por las universidades y los realmente ocupados. Estos últimos fluctuaron entre 59 y $100 \%$, siendo el promedio de $75,5 \%$.

\section{Número de médicos especialistas reconocidos o en formación}

En base a datos confiables, el número de especialistas se ha podido establecer desde el año 1996, cuando existían 15.563 médicos activos según Goic $^{13}$, de los cuales, de acuerdo a la estimación del Colegio Médico ${ }^{14}, 43 \%$ se definían como médicos generales, y por tanto, el $57 \%$ restante $(8.870$ médicos) podía ser considerado especialista. Sin embargo, 8 años después (2004) la cifra total de especialistas certificados por CONACEM desde 1984 era de tan sólo 8.356 médicos. Esta discordancia se puede explicar por dos razones: por un lado, en los servicios de salud existía casi $20 \%$ de médicos contratados como especialistas pero sin certificación ${ }^{15}$, y por otro, muchos especialistas certificados gracias a programas universitarios, no se inscribían necesariamente en $\mathrm{CONACEM}^{7}$.

De acuerdo a los estudios de nuestro grupo ${ }^{15}$, la cifra de especialistas en el año 2004 era de 14.306 (56\%) de un total de 25.542 médicos catastrados en el país, hubiesen sido certificados por las universidades, CONACEM, o extranjeros con reválida ${ }^{16}$. Sin embargo, de acuerdo a los registros de CONACEM hasta el año 2010, el número de especialistas certificados en el país alcanzaba a 11.389 , diferencia que de nuevo se explica por la existencia de especialistas no certificados aún, en espera de hacerlo o por estar acreditados por universidades nacionales o extranjeras ${ }^{7,15}$.

A esta estimación global al año 2010, es preciso agregar los 218 especialistas formados por los programas analizados, que al haber ingresado en

Tabla 1. Médicos ingresados y obligación de retorno a programas de formación de especialistas período 2007-2010

\begin{tabular}{|c|c|c|c|c|c|}
\hline Programa / línea de formación & 2007 & 2008 & 2009 & 2010 & Total \\
\hline Médicos EDF & 133 & 153 & 163 & 143 & 592 \\
\hline Médicos APS (3 años) & 13 & 56 & 78 & 72 & 219 \\
\hline Médicos formación directa SS & 45 & 128 & 150 & 259 & 582 \\
\hline Médicos especialistas básicos APS (6 años) & 0 & 31 & 74 & 102 & 207 \\
\hline Total & 191 & 368 & 465 & 576 & 1.600 \\
\hline \multirow{2}{*}{$\begin{array}{l}\text { Con período asistencial } \\
\text { obligatorio (PAO) }\end{array}$} & 58 & 184 & 228 & 331 & 801 \\
\hline & 30,4 & 50,0 & 49,0 & 57,5 & 50,1 \\
\hline
\end{tabular}

Tabla 2. Diferencia entre cupos ofertados por el MINSAL/SS y ocupados por médicos

\begin{tabular}{|lccc|}
\hline Programa / línea de formación & Ofertados & Ingresados & Diferencia \% \\
\hline Médicos EDF & 958 & 592 & 61,8 \\
Médicos APS (3 años) & 369 & 219 & 59,3 \\
Médicos formación directa SS & 582 & 582 & 100,0 \\
Médicos especialistas básicos APS (6 años) & 211 & 207 & 98,1 \\
\hline Total & 2.120 & 1.600 & 75,5 \\
\hline
\end{tabular}


Formación de médicos especialistas en Chile - O. Román et al

el 2007, ya han cumplido tres años y debieran estar en proceso de certificación. Si extrapolamos los resultados del programa de especialización hasta el 2013, llegamos a una cifra aproximada de 1.600 nuevos especialistas (Tabla 1 ).

\section{Distribución en los sistemas público o privado}

No se conoce un catastro exacto de los especialistas en el sistema privado. Sólo podemos establecer que de los 14.306 reconocidos en el año 2004, sólo 6.428 estaban contratados en los servicios de salud, lo que equivale a $45 \%$, y por tanto, el resto en el sistema privado u otras instituciones públicas.

La tendencia preponderante en la actualidad, tanto de médicos como estudiantes de medicina, es preferir el sistema privado ${ }^{12,17-19}$. En consecuencia, la política pública de recursos humanos debería orientarse a la captación y retención de especialistas. Al respecto, de los 4 programas o líneas de formación existentes, sólo los de APS municipal de tres años y los de formación directa por los SS tienen PAO o período asistencial obligatorio posterior. Al aplicar este destino a los médicos ingresados al proceso de formación, sólo 58 de los 191 del año 2007 tienen esa obligación (30,4\%) y en los años sucesivos ella llega a 50\% (Tabla 1), fluctuando entre 3 y años.

\section{Cargos $u$ horas especialista en el sistema público}

De acuerdo a los registros de la División de Gestión y Desarrollo de las Personas del MINSAL existía en el año 2006 una carencia de especialistas en el sistema público de 888 médicos de jornada completa (44 hrs) ${ }^{18}$. En el año 2008, el estudio del Banco Mundial ${ }^{20}$, demostró que en las 26 especialidades más significativas, la brecha de horas especialistas en el sistema público era de 45.749 hrs/sem., lo que equivalía a 1.039 médicos.

\section{Tipo de especialidades}

En el año 2004 nuestro grupo ${ }^{18}$ registró 3.688 médicos en las 5 especialidades primarias básicas, lo que correspondía a 63\% del total de especialistas, y 37\% restante, a los especialistas primarios (2.205 médicos). En el estudio actual, la tendencia porcentual de formación es de $47 \%$ en las especialidades básicas y 53\% en las primarias (Tablas 3 y 4). En las especialidades básicas, la Medicina Interna ha pasado a ser la de mayor preferencia, seguida por Pediatría. Además aparece un significativo aumento de la Psiquiatría, que ha sobrepasado a la Cirugía General y a la Obstetricia.

En las especialidades primarias, los primeros lugares los ocupan Anestesia, Radiología, Ortopedia, Traumatología y Medicina Familiar, siendo los menos frecuentes Neurocirugía, Fisiatría y Urología, además de una miscelánea de otras especialidades.

\section{Distribución regional de los especialistas}

La distribución regional de los médicos en proceso de formación se correlaciona con la demografía regional, en especial respecto a la población beneficiaria del sistema público. Así, las regiones V, VIII y Metropolitana son las que presentan mayores ingresos a programas de especialización, siendo inferiores en las regiones I, III, VI, VII, y las 3 más australes.

Tabla 3. Médicos ingresados a programa de especialidades básicas, según línea de formación período 2007-2010

\begin{tabular}{|lcccc|}
\hline Especialidad & EDF & \multicolumn{2}{c|}{ Programas de especialización } \\
Medicina Interna & 80 & Directa SS & Esp. básicos APS & Total \\
\hline Pediatría & 49 & 86 & 39 & 205 \\
\hline Psiquiatría & 45 & 37 & 51 & 137 \\
Cirugía General & 46 & 48 & 32 & 125 \\
\hline Obstetricia Ginecología & 15 & 51 & 0 & 97 \\
Total & 235 & 10 & 41 & 66 \\
\hline
\end{tabular}


Tabla 4. Ingresados a especialidades primarias según líneas de formación, período 2007-2010

\begin{tabular}{|lcccc|}
\hline Especialidad & EDF & APS $\mathbf{3}$ años & Directa SS & Total \\
\hline Anestesia & 46 & 2 & 68 & 116 \\
\hline Radiología & 33 & 0 & 56 & 89 \\
\hline Ortopedia y Traumatología & 49 & 2 & 35 & 86 \\
\hline Medicina familiar & 25 & 39 & 9 & 73 \\
\hline Neurología adulto & 44 & 0 & 15 & 59 \\
Oftalmología & 27 & 0 & 20 & 47 \\
\hline Dematología & 19 & 0 & 22 & 41 \\
\hline Anatomía patológica & 17 & 3 & 9 & 29 \\
\hline Otorrinolaringología & 20 & 0 & 7 & 27 \\
Neurocirugía & 13 & 0 & 10 & 23 \\
\hline Fisiatría & 14 & 0 & 6 & 20 \\
\hline Urología & 10 & 0 & 7 & 17 \\
\hline Otras & & 46 & 264 & 83 \\
\hline Total & 317 & & & 710 \\
\hline
\end{tabular}

\section{Costo de los programas}

La formación de cada especialista tiene dos elementos de costo: uno anual, referido a los aranceles que devengan las universidades formadoras, que alcanza a $\$ 2.800 .000$ el año 2010 y el segundo, un aporte mensual de $\$ 850.000$ a los SS para el reemplazo de los médicos en formación. En la Figura 1 se muestra el aumento de recursos presupuestarios entregados entre 2007 y 2010.

\section{Retención de los especialistas en el sistema público}

Desde 1997 a 2007, el estudio realizado por MINSAL $^{9}$ ha demostrado que la mantención de cargos en el sistema público es relativamente significativo, pues desde cerca de $70 \%$ en los 3 primeros años se reduce a $60 \%$ en los 4 años siguientes (Figura 2).

\section{Oferta de cupos de especialización por universidades}

$\mathrm{Al}$ año 2010 la oferta de cupos de especialización de 11 universidades llegó a 777, siendo la Universidad de Chile la que ofreció más vacantes (47\%). Le siguen la Pontificia Universidad Católica de Chile, Universidad de Concepción y Universidad de Santiago, con $10 \%$ cada una. El resto ofreció menores porcentajes.

\section{Discusión}

El aumento de oportunidades para la especialización médica que hemos descrito se ha debido a la conjunción de dos acciones fundamentales: los esfuerzos programáticos universitarios y el financiamiento necesario otorgado por MINSAL ${ }^{9}$. El acuerdo establecido entre Ministerio de Salud y la ASOFAMECH en representación de las universidades del país, tanto públicas como privadas, ha permitido el avance descrito, que está siendo respaldado por disposiciones legales y reglamentarias vigentes que regulan las cuatro líneas o caminos de especialización existentes $s^{5,6,8,9}$.

Los cupos tomados u ocupados por los médicos lo han sido en base a concursos amplios de carácter nacional o locales organizados por los propios servicios de salud. La duración de estos programas ha sido la reglamentaria y legal, siendo interesante resaltar que el PAO (período asistencial obligatorio posterior) rige sólo para los médicos APS y de los SS. En esos casos, las instituciones contratantes, tanto municipalidades como servicios de salud, pueden influenciar el tipo de especialización, de acuerdo a las brechas y necesidades reales que tengan, especialmente a nivel regional.

En el proceso que analizamos, han participado varias universidades nacionales, tanto públicas 


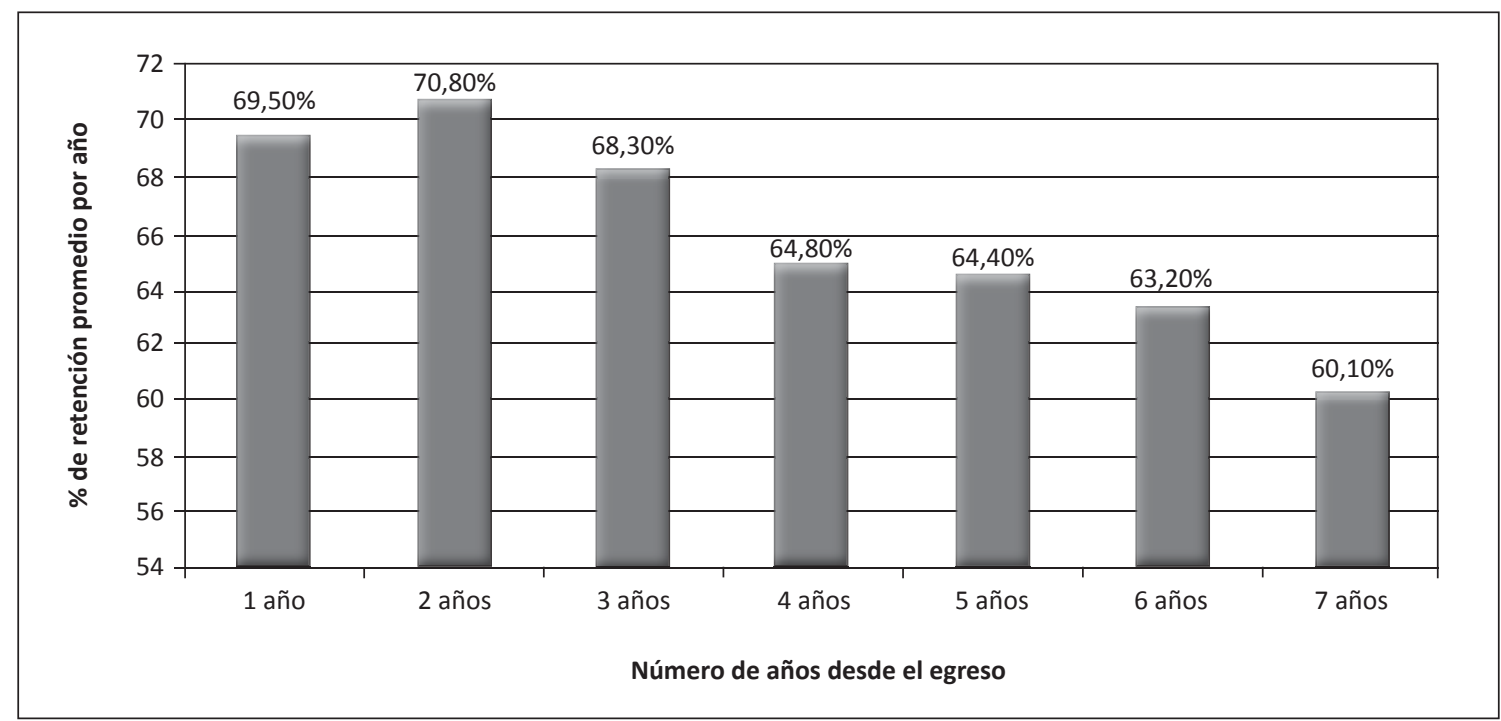

Figura 1. Incremento presupuestario (M\$) de los Programas de Especialidades para Médicos. Período 2007-2010.

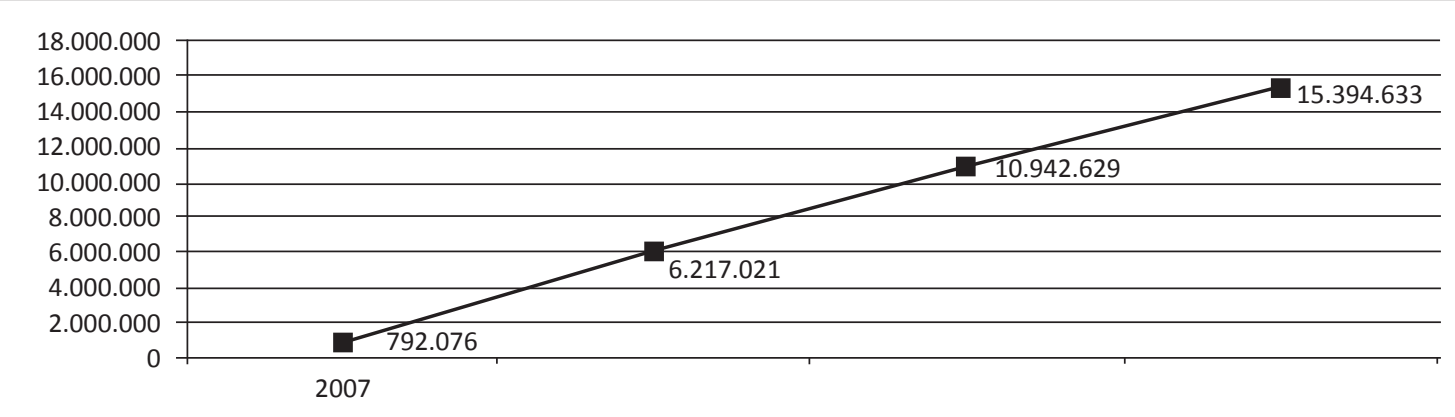

Figura 2. Promedio de retención de los médicos becados Minsal en los Servicios de Salud a un número dado de años desde el egreso (cohorte 1997-2007).

como privadas, lo que revela la presencia de una capacidad formadora importante en el ámbito del post-título médico.

Sin embargo, es preciso reconocer que aún persisten problemas a resolver, uno de los cuales, tal vez el más importante es concordar las normas que regulan la asignación y uso de los campos clínicos para desarrollar los programas de pre y post-grado.

En relación al destino futuro de estos especialistas, en particular a su mantención en el sistema público, hemos observado que la continuidad en éste ha sido alta en los años precedentes (Figura 2). Surge de inmediato la interrogante; ¿cuál va ser el destino futuro de este importante número de especialistas en formación? Su permanencia en el sistema público, en gran medida va a depender de las remuneraciones y particularmente de la existencia de tecnologías, insumos y personal de colaboración necesarios para el desarrollo de su labor especializada, según se desprende de diversos estudios y comunicaciones científicas nacionales ${ }^{15,17-21}$. Esta necesidad ha sido reconocida por las autoridades y se espera que en el presupuesto se incorporen recursos suficientes que permitan la retención o atracción de médicos especialistas a los diversos SS, especialmente a aquellos de zonas extremas y alejadas del país.

En el aumento de oportunidades de especia- 
lización destaca el advenimiento de los denominados especialistas básicos, que son especialistas primarios que se forman en forma mixta en atención primaria y secundaria. Su origen nació en gran medida en la necesidad de reforzar la capacidad resolutiva clínica de la acción médica de la APS. Si bien se discutió al comienzo la pertinencia de formar especialistas en la atención primaria, entendida y definida como propia del quehacer de médicos generales, los acuerdos sucesivos entre el MINSAL y las universidades, especialmente la Universidad de Chile, permitió al Ministerio de Salud iniciar este programa en el año $2008^{11,12}$. Su evaluación deberá esperar 6 años, que es el tiempo establecido para completar la especialización.

La urgencia en incrementar el número de médicos especialistas se ha basado en la necesidad de cerrar las brechas de atención especializada en el país, determinadas por los estudios de la Secretaría Técnica del AUGE (2004) y del Banco Mundial. $(2009)^{16,19}$. La falta de especialistas señalada en estos estudios contrasta con la existencia en el país de un franco predominio de médicos especialistas sobre los generales, que alcanzaban al $56 \%$ en el país en el año 2004 y al 64\% en el total del sistema público y al $81 \%$ en los servicios de salud ${ }^{15,18}$. Esta última cifra se elevó al $85 \%$ en el año $2008{ }^{17}$. Sin embargo, es preciso reconocer que a pesar que en los años 2004 y 2008 los especialistas básicos tenían un número y distribución regional satisfactoria ${ }^{15,18}$, los especialistas primarios y los sub-especialistas mostraban brechas de significación ${ }^{18,19}$.

Sin embargo, de acuerdo a nuestros actuales datos, los futuros especialistas primarios tendrán un número superior al de los básicos, lo que sugiere que podrán cerrarse algunas brechas correspondientes.

Respecto al interés de los médicos por el tipo de especialidad escogida, dado que el ingreso ha sido opcional, se ha observado una predilección mayor por Medicina Interna y Psiquiatría en las básicas y por Anestesiología y Radiología en las primarias.

Otro aspecto importante de señalar es que el aumento de médicos especialistas ha sido proporcional al incremento de la población profesional médica del país, la que ha crecido en forma significativa en los últimos años, como lo señala la Tabla 5, que muestra la evolución de matrícula y egreso de médicos entre 2002 y 2009 en las universidades chilenas ${ }^{17}$. Se podría pensar que el aumento de oferta de médicos pudiera canalizarse hacia médicos generales que vayan a reforzar la APS, fundamental en una política sanitaria de diagnóstico precoz y prevención de las patologías más importante y frecuentes. Pero los estudios sociológicos de los egresados y estudiantes de medicina señalan que el camino preferido de ellos está orientado hacia la especialización inmediata y tecnológicamente eficiente ${ }^{20}$. Es preciso aceptar entonces que el incremento del número de médicos va a ir paralelo a un aumento del subproducto "Médicos especialistas", porque es preciso reconocer una creciente necesidad de ellos en una sociedad que crece en desarrollo y cultura sanitaria y que por tanto los reclama.

Lo anterior no elimina la necesidad de conciliar el interés de una atención rápida y eficiente en la primera línea, que forma la APS, con aquella

Tabla 5. Evolución matrícula y egreso universidades chilenas médicos período 2002 - 2010

\begin{tabular}{|cccc|}
\hline Año & Matrícula primer & Total & Egresados \\
\hline 2002 & 1.255 & 6.691 & 738 \\
\hline 2003 & 1.429 & 7.353 & 724 \\
\hline 2004 & 1.599 & 8.147 & 919 \\
\hline 2005 & 1.689 & 8.783 & 925 \\
\hline 2006 & 1.634 & 9.503 & 911 \\
\hline 2007 & 1.711 & 10.060 & 939 \\
\hline 2008 & 1.786 & 10.855 & 888 \\
\hline 2009 & 1.827 & 11.425 & 1.061 \\
\hline
\end{tabular}

Fuente: Cuadro elaborado en base a información publicada por el Consejo Superior de Educación, abril 2010. 
especializada, basada en evidencia científica, que sea eficaz y tecnológicamente eficiente a nivel secundario y terciario.

Para lograr aquel objetivo, basado en una política sanitaria científica y ética, se requiere aunar esfuerzos, acuerdos y coordinación, con perspectiva futura, entre las instituciones involucradas, Ministerio de Salud, universidades, Colegio Médico y municipalidades.

\section{Referencias}

1. Diario El Mercurio. Salud ha cubierto tan sólo el $9 \%$ de las horas de especialidades médicas. 12 octubre 2008 .

2. Diario La Tercera. Consultas médicas de especialidades crecieron 53,5\%. 2 mayo 2010.

3. Diario El Mercurio. Déficit de médicos especialistas es de $40 \%$ en hospitales y lo agrava la expansión de Fonasa. 10 mayo, 2010.

4. Semanario Estrategia. US $\$ 42$ millones al año costaría al Minsal cubrir el déficit de médicos especialistas. Junio, 2010.

5. Ministerio de Salud, Universidades y Colegio Médico de Chile. Proyecto-Programa de política de formación de especialistas en Chile. Abril 2010.

6. Escuela de Post-Grado. Facultad de Medicina Universidad de Chile, Normas reglamentarias sobre programas de especialidades conducentes a título. 1996-1997.

7. Comision Nacional de Certificacion de Especialidades Medicas (CONACEM). Registro de certificación de Especialidades Médicas. Vida Médica 2004; 56: 18-9.

8. Diario Oficial. Ley 19664. Establece normas especiales para profesionales funcionarios que indica en los Servicios de Salud y modifica la Ley 15076. Febrero, 2000.

9. Ministerio de Salud. Unidad de Estudios de la Subsecretaría de Redes Asistenciales, cohortes 1997 - 2007. Exposición estudio Banco Mundial, mayo 2010.

10. Ipinza M. El modelo de atención integral de salud, base doctrinaria del programa de formación de médicos especialistas básicos para la atención primaria de salud. Cuad Med Soc (Chile) 2: 132-42.

11. Escuela de Medicina. Universidad de Chile. Historia de la Medicina en Chile. www: escuela med.puc.cl/publ/
Historia Medicina/Indice.

12. Román O, Pineda S, Señoret M. Perfil y número de médicos generales que requiere el país. Rev Med Chile 2007; 135: 1209-15.

13. Goic A. Disponibilidad de médicos en Chile y su proyección a mediano plazo. Cinco años después. Rev Med Chile 1999; 127: 1183-8.

14. Montero J, Muñoz F, Rosselot E, Valdivieso V, Barba R. La medicina general en la organización médica actual. Rev Med Chile 1996; 124: 1006-14.

15. Román O, Acuña M, Señoret M. Disponibilidad de médicos en Chile al año 2004. Rev Med Chile 2006; 134 1057-64.

16. Rosselot E, Mateluna E, Estevez A, Radrigán ME. Acreditación de título de profesionales e instituciones formadoras: el caso de Chile. Rev Med Chile 1998; 126: 715-24.

17. Román O. Evolución de la oferta de médicos. Chile 1993-2008. Rev Med Chile 2010; 138: 22-8.

18. Román $\mathrm{O}$, Señoret M. Estado actual de las especialidades médicas en Chile; realidad en el sistema público no municipalizado. Rev Med Chile 2008; 136: 99-106.

19. Banco Mundial y Gobierno de Chile. Estudio de brechas de oferta y demanda de médicos especialistas en Chile. Informe. Diciembre 2009.

20. Ministerio de Salud. Unidad de estudios de recursos humanos. Motivación y opciones laborales de los médicos especialistas. Síntesis del estudio Sociológico. Mayo 2010.

21. González F. Implementación del plan Auge en pacientes con Insuficiencia renal crónica. Rev Med Chile 2003, 131: 545-51.

22. Armas Merino R, Goic A. Informe sobre la situación actual de la Educación Médica en Chile. Comité de Educación Superior. Academia de Medicina. 2008. Publ. Academia de Medicina.

23. Torres C, Solimano G. Formación de médicos especialistas en el contexto de la reforma de salud EDE Chile. Monografía 2003. Universidad de Chile. Escuela de Salud Pública.

24. Enríquez O. Política Nacional de formación de médicos especialistas: un proceso en desarrollo. Editorial. Cuad Med Soc (Chile) 2010; 2: 81-2. 